\title{
Sunspot Proper Motion and Flare Frequency
}

\author{
G. Csepura ${ }^{1}$, L. Györi ${ }^{1}$, A.A. Galal ${ }^{2}$ \\ 1 Heliophysical Observatory of the Hungarian Acad. Sci., H-4010 Debrecen, \\ P.O. Box 30, Hungary \\ 2 National Research Institute of Astronomy and Geophysics, Helwan, Cairo, Egypt
}

Flare activity of solar active regions is generally believed to depend on a sheared configuration of magnetic fields (Hagyard et al. 1984). There are cases when the shear necessary for a flare can be attributed to the emergence of a new flux in the spot group (Wang 1992). But, perhaps, a newly born active region can also influence the magnetic field configuration in a nearby active region (Poleto et al. 1993, Gesztelyi et al. 1993). In this paper we are interested primarily in the influence of a newly emerging spot group on a nearby one.

The three neighbouring active regions NOAA AR 6412(B-C), 6413(A) and 6415(D) have been studied between 13-22 December 1990. White-light pictures for studying sunspot proper motion and area evolution were taken at Gyula Observing Station (Hungary), Debrecen Heliophysical Observatory (Hungary) and Helwan Observatory (Egypt). Times and positions of the flares were taken from the Solar Geophysical Data (No. 558, part 1, February 1991).

Interesting events have been found on December 16 and 18, 1990.

1990 December 16: This was the day when the emergence of the newly born region $\mathrm{D}$ began (Fig. 1). Up to this day the area of the active region $\mathrm{B}-\mathrm{C}$ continuously increased but afterwards it began to decrease (Fig. 1.Iv). From this day on, the spots $\mathrm{B} 1$ and $\mathrm{B} 2$ in the active region $\mathrm{B}-\mathrm{C}$ started a relative revolution around each other and in the same active region, there was a sudden increase in the relative revolution of spots C1 and C2 (Fig. 1.I, 1.III). Up to December 16, the occurrence of flares in the active region B-C was scattered over the whole region but on this day they were concentrated between $\mathrm{B}$ and $\mathrm{C}$ and increased, while, contrary to this, the daily flare frequency in these active regions was found to have a local minimum (Fig. 1.I, 1.II).

1990 December 18: On this day, group $D$ reached its maximal area, and at the same time, the total umbral area had its maximal value also (Fig. 1.Iv). It is interesting that up to this time the umbral areas of the spots had practically not changed (except group D) but on this day there was a slight increase in their values and after this day the previous values were restored. The positions of the flares after this day is shifted to group D (Fig. 1.I, 1.II). As was found on December 16, the rate of the relative revolution of spots C1 and C2 increased on this day, too (Fig. 1.I, 1.11I). The above events can probably be explained by the fact that this was the day when group D started to decline. 
Considering the above coincidences, we suggest that the emergence or the fast decline of an active region can exert an influence on another active region in its vicinity.

Acknowledgement: This work was supported by the Hungarian Foundation for Scientific Research under grant No. OTKA 007422.
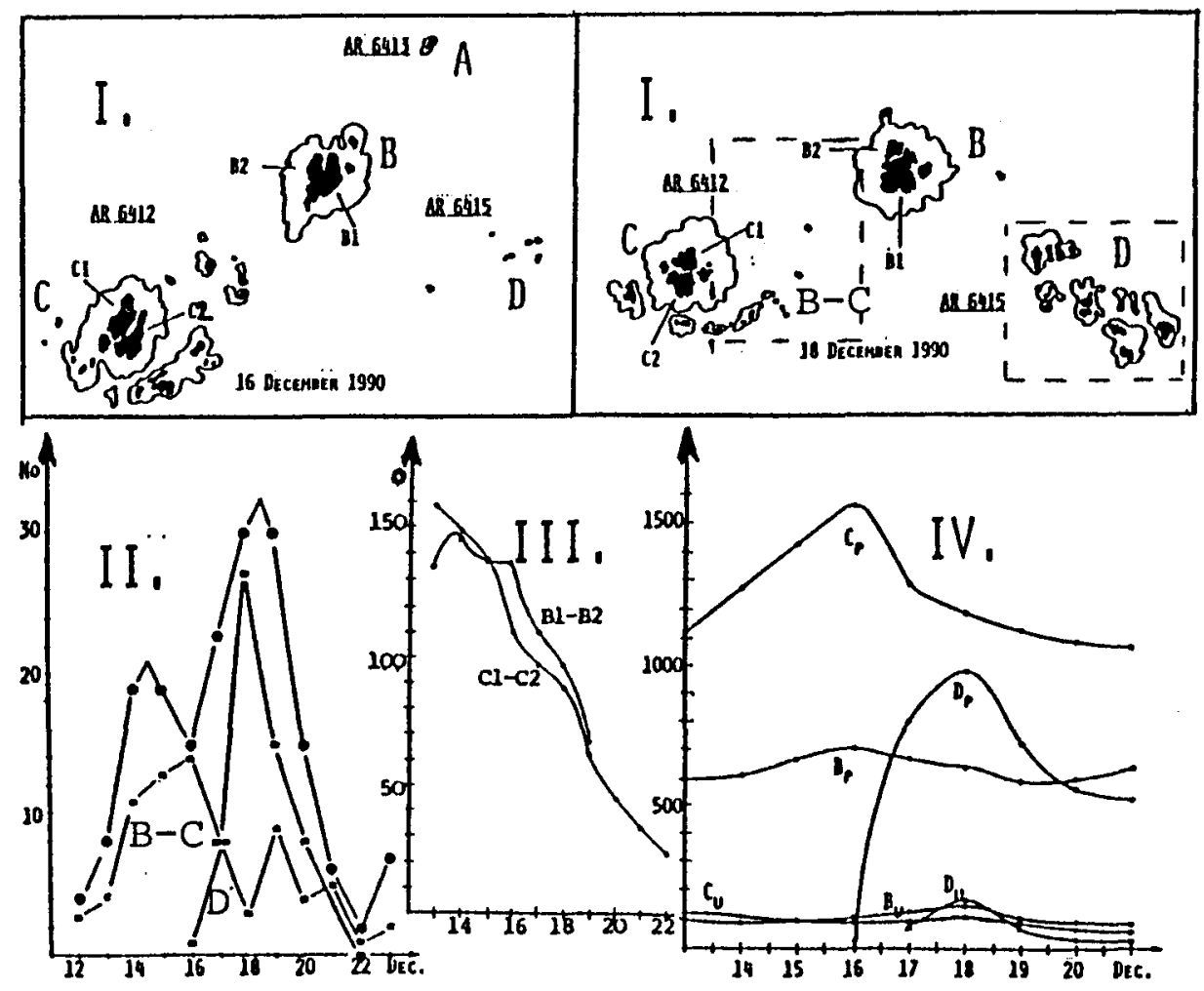

Fig. 1. I. Development of the sunspot groups. II. Unmarked curve shows the daily numbers of flares. The other two curves show the daily number of flares in the two flare centra (B-C and D). III. The rate of the daily relative revolution of spots B1 and B2 and spots C1 and C2. Iv. Development of the total umbral and penumbral area of groups B, C and D, expressed in millionths of the Sun's visible hemisphere.

\section{References}

Hagyard M.J., Smith J. Jr., Teuber D., West E. A., 1984, Solar Phys. 91, 115

Wang H., 1992, Solar Phys. 140, 85

Poletto G., Gry G.A., Machado M.E., 1993, Solar Phys. 144, 113

van Driel-Gesztelyi L., Csepura L., Nagy I., Gerlei O., Schmieder B., Rayrole J., Demoulin P., 1993, Solar Phys. 145, 77 